\title{
The Tumor Growth Paradox and Immune System-Mediated Selection for Cancer Stem Cells
}

\author{
Thomas Hillen • Heiko Enderling • \\ Philip Hahnfeldt
}

Received: 24 July 2012 / Accepted: 9 November 2012

(C) Society for Mathematical Biology 2012

\begin{abstract}
Cancer stem cells (CSCs) drive tumor progression, metastases, treatment resistance, and recurrence. Understanding CSC kinetics and interaction with their nonstem counterparts (called tumor cells, TCs) is still sparse, and theoretical models may help elucidate their role in cancer progression. Here, we develop a mathematical model of a heterogeneous population of CSCs and TCs to investigate the proposed "tumor growth paradox"-accelerated tumor growth with increased cell death as, for example, can result from the immune response or from cytotoxic treatments. We show that if TCs compete with CSCs for space and resources they can prevent CSC division and drive tumors into dormancy. Conversely, if this competition is reduced by death of TCs, the result is a liberation of CSCs and their renewed proliferation, which ultimately results in larger tumor growth. Here, we present an analytical proof for this tumor growth paradox. We show how numerical results from the model also further our understanding of how the fraction of cancer stem cells in a solid tumor evolves. Using the immune system as an example, we show that induction of cell death can lead to selection of cancer stem cells from a minor subpopulation to become the dominant and asymptotically the entire cell type in tumors.
\end{abstract}

Keywords Cancer stem cells · Cell death · Immune system · Integro-differential equation model $\cdot$ Geometric singular perturbation analysis

\footnotetext{
T. Hillen

Centre for Mathematical Biology, Mathematical and Statistical Sciences, University of Alberta, Edmonton T6G 2G1, Canada

e-mail: thillen@ualberta.ca

H. Enderling · P. Hahnfeldt (凶)

Center of Cancer Systems Biology, Steward Research \& Specialty Projects Corp., St. Elizabeth's

Medical Center, Tufts University School of Medicine, Boston, MA 02135, USA

e-mail: philip.hahnfeldt@tufts.edu

H. Enderling

e-mail: heiko.enderling @ tufts.edu
} 


\section{Introduction}

Cancer has been characterized as a collection of diseases described by uncontrolled growth of cells and development of a tumor that invades the tissue of origin and distant organs. Cancer cells are generally assumed to have acquired a series of mutations to express a phenotype with the so-called six "Hallmarks of Cancer": evading apoptosis, limitless replicative potential, self-sufficiency in growth signals, insensitivity to antigrowth signals, sustained angiogenesis, tissue invasion and metastasis, plus four recently identified additional traits of deregulation of cellular energetics, avoidance of immune destruction, genome instability, and tumor promoting inflammations (Hanahan and Weinberg 2000, 2011). One long-held paradigm in cancer research has been that once a cancer cell has successfully developed it will inevitably form a frank tumor. Recent research suggests, however, that early tumor growth and progression is challenged by intrinsic and environmental bottlenecks such as the angiogenic switch (Folkman and Hanahan 1991; Almog et al. 2009; Hahnfeldt et al. 1999), immune surveillance (Dunn et al. 2002; Teng et al. 2008; D'Onofrio 2005), or the epithelial-mesenchymal transition (EMT) (Hanahan and Weinberg 2011). Furthermore, it is increasingly argued that only a small subset of cancer cells is intrinsically able to initiate and repopulate the tumor. Distinct properties of these so-called cancer stem cells (CSCs) are longevity or even immortality, self-renewal, unlimited proliferation and the ability to produce more such cancer stem cells as well as non-stem cancer cells (or tumor cells, TCs).

In this paper, we develop an integro-differential equation model that is based on the same biological assumptions as the agent-based model of Enderling et al. (2009). We reduce this integro-differential equation model to a simple nonlinear ordinary differential equation (ODE), which describes the time evolution of CSC and TC. We show, using the geometric singular perturbation theory (Hek 2010), that the simple ODE model is sufficient to explain the tumor growth paradox. The simplicity of the model underlines the often-neglected pivotal role of intrinsic cell properties and cell-cell interactions in tumor growth and morphological evolution. The presented approach should be widely applicable to solid, avascular tumors in any organ. We propose that the tumor growth paradox within the cancer stem cell hypothesis is a basic kinetic feature, which has profound implications on treatment design. As applied specifically to the immune response, we further show that selection for the (more resistant) CSCs will take place, inevitably leading to an increasing CSC fraction in in-vivo tumors.

\subsection{Cancer Stem Cells}

The first evidence of a CSC subpopulation surfaced for leukemia, when it was discovered some, but not all, cancer cells where able to initiate and reinitiate tumors of the blood (Lapidot et al. 1994; Bonnet and Dick 1997). Recently, CSCs have also been associated with solid tumors, for example, of the breast, brain, prostate, and colon (Al-Hajj et al. 2003; Dick 2003; Singh et al. 2003, 2004; Fioriti et al. 2008; Maitland and Colling 2008; Todaro et al. 2007; Cammareri et al. 2008). If a subpopulation of CSCs exists in the tumor, then the others by exclusion are nonstem TCs, which by definition have only limited replicative potential and a limited lifespan. TCs 
are produced during asymmetric stem cell division, during which the stem cell maintains its property and the daughter cell is fated to mature (Morrison and Kimble 2006; Reya et al. 2001; Dingli and Michor 2006). Although different differentiation levels of nonstem TC with respective terminologies are observed (transient cells, transit amplifying cells, progenitor cells, differentiated cells, mature cells), herein we combine all cancer cells that lack stemness as tumor cells (TC).

Although CSC are necessary for tumor development, tumor progression is dependent on many additional factors including oxygen and nutrient availability (Folkman 1971; Naumov et al. 2006), the tumor microenvironment (Barcellos-Hoff 2001; Gatenby and Gillies 2008), immunosurveillance (Dunn et al. 2002; D'Onofrio 2005) and even the nonstem TC population (Prehn 1991; Enderling et al. 2009; Morton et al. 2011). The complex dynamics emerging from cell-cell and cellmicroenvironment interaction are difficult to predict and novel experimental tools are only just emerging. Therefore, mathematical models and agent-based computer models are increasingly utilized to find core mechanisms that drive tumor development and progression (Alarcon et al. 2006; Anderson et al. 2006; Conde-Ramis et al. 2008; Enderling et al. 2008; Gevertz and Torquato 2006; Mallet and De Pillis 2006; Ribba et al. 2004; Smallbone et al. 2007; Quaranta et al. 2005, 2008; Liu et al. 2007).

\subsection{Individual Based Modeling}

Enderling et al. (2009) have previously developed a single-cell-focused agent-based model to simulate the dynamics of CSCs and TCs and their competition for space in-silico. In this model, cells live on a square lattice, and can only migrate or proliferate if any of their neighboring lattice sites are vacant. When all adjacent grid points are occupied the cells are forced into quiescence. In the early growth phases, TCs quickly outnumber CSCs, causing the CSCs to become quiescent. Tumor progression dynamics then solely depend on progeny TC that eventually exhaust their proliferation capacity, thereby stalling tumor growth. For tumor progression, CSCs need to reenter the cell cycle and produce more CSCs that opportunistically migrate away to form new tumor clusters nearby. Simulations of the agent-based model revealed that an increasing rate of spontaneous cell death in TC shortens the waiting time for CSC proliferation and migration, and thus facilitates tumor progressiona phenomenon we call the tumor growth paradox. The emergence of many "selfmetastatic" (Norton 2005; Enderling et al. 2009b) clusters in the vicinity of each other drives both tumor expansion and an aggressive tumor morphology. Figure 1 shows simulation results of how the total number of cancer cells increases with increased cell death, and some representative morphologies of the emerging tumors.

\subsection{Mathematical Models of Stem Cells and Cancer}

In the past few years, there has been a surge of mathematical models on stem cells and cancer. Marciniak-Czochra et al. (2009) studied several models for stem cells and a series of transient cell compartments with focus on the role of regulatory feedback mechanisms that are necessary for homeostasis and efficient repopulation. While the 


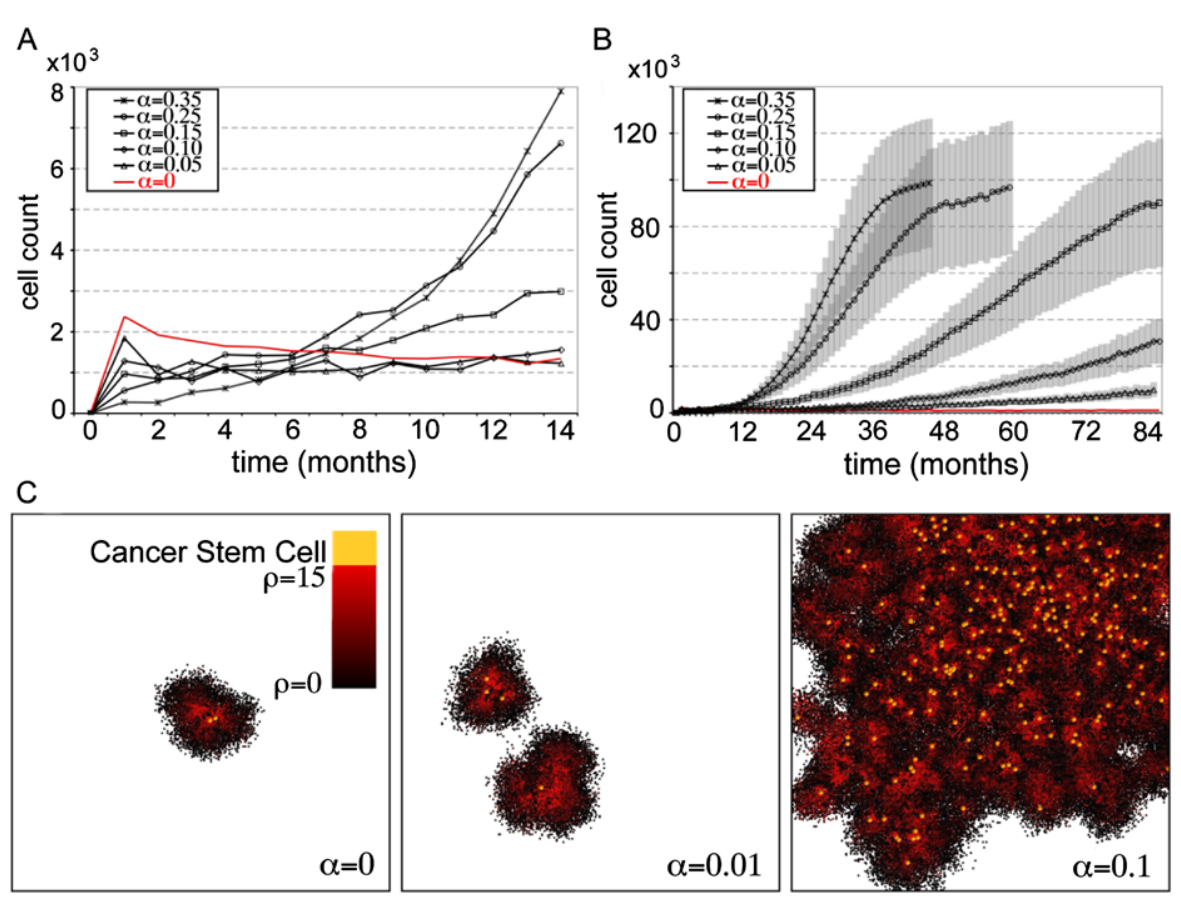

Fig. 1 Simulations of tumor growth by the cellular automaton model from Enderling et al. (2009). The growth dynamics of a tumor without cell death $\left(p_{\alpha}=0\right.$, red $)$ is compared to tumor growth with $p_{\alpha}=0.05,0.1,0.15,0.25,0.35$ for (A) 15 months and (B) 85 months ( $\rho=15, \mu=5$ ). Initially, the tumor growth is inhibited with increasing cell death rate. After $t=7-15$ months, a complete reversal of the cell death effect can be observed. (C) Representative simulations of tumors developing with spontaneous cell death rates $p_{\alpha}=0,0.01,0.1$ after $t=8$ months $(\rho=15, \mu=15)$ (Color figure online)

works of Ganguli and Puri (2006) and Sole et al. (2008) focused on a model of transforming normal stem cells to cancer stem cells through a series of mutations, Dingli and Michor (2006) identified the dominating role of CSC in driving tumor progression with the hallmark conclusion that "Successful therapy must eradicate cancer stem cells." Enderling et al. (2009) developed a model of cancer stem cell-driven tumor growth in which the contribution of different cell kinetic parameters to overall tumor progression was rigorously investigated. In a series of papers, Wise et al. (2008) and Youssefpour et al. (2012) developed a very detailed model for tumor growth that includes many physical aspects of tumor interaction with the host tissue. This model can readily be extended to include stem cell dynamics.

As evident in the above reviewed literature, different models and modeling techniques can be used to simulate cancer stem cell kinetics. The models, however, differ in the formal description of how (cancer) stem cells divide. Indeed, their assumptions cover the full range of possibilities, i.e., the result of a CSC division can either be (i) two CSC (symmetric division), (ii) one CSC and one TC (asymmetric division), or (iii) two TC (symmetric commitment) (Fig. 2). Despite these several possibilities, however, we show in the Appendix that under very broad conditions the two major cell division strategies that have evolved lead to models that are fundamentally equivalent. 


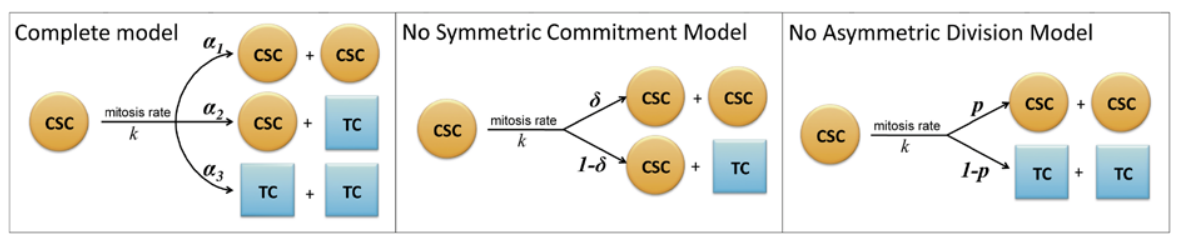

Fig. 2 Schematic representation of three conceptually distinct models of CSC division fates. CSC divide with rate $k$, and produce either two CSC, two TC, or one CSC and one TC

\section{A Mathematical Model of Cancer Stem Cell-Driven Solid Tumor Growth}

To complement the computational model from Sect. 1.2, we develop a mathematical model in form of integro-differential equations, that consists of cancer stem cells (CSCs) and nonstem tumor cells (TCs). We use the following biological assumptions as laid out in the agent-based model by Enderling et al. (2009):

Assumptions for Cancer Stem Cells CSCs are immortal (rate of cell death $\alpha=0$ ) and have an infinite proliferation capacity. A CSC can give rise to two CSCs (symmetric division), or one CSC and one TC (asymmetric division), in line with the 'No Symmetric Commitment Model' discussed in the Appendix.

Assumptions for Non-stem Cancer Cells TC proliferation always results in two TCs, and TCs have a positive probability of cell death reflecting exhaustion of proliferation potential as well as spontaneous death due to genomic instability $(\alpha>0)$.

Simulations of the agent-based model (Enderling et al. 2009) revealed a tumor growth paradox. With increased cell death in the TC compartment tumor growth is accelerated. We define this paradox in the mathematical model as follows.

Definition 2.1 Let $P_{\alpha}(t)$ for times $t \geq 0$ denote a tumor population with a spontaneous death rate $\alpha$ for TC. The population exhibits a tumor growth paradox if there exist death rates $\alpha_{1}<\alpha_{2}$ and times $t_{1}, t_{2}$, and $T_{0}>0$ such that

$$
P_{\alpha_{1}}\left(t_{1}\right)=P_{\alpha_{2}}\left(t_{2}\right) \quad \text { and } \quad P_{\alpha_{1}}\left(t_{1}+T\right)<P_{\alpha_{2}}\left(t_{2}+T\right) \quad \text { for }\left(0<T<T_{0}\right) .
$$

To describe tumor growth on the population level, we use reaction-birth processes (Greese 2006) and develop a mean-field integro-differential equation system. A reaction-birth process refers to a stochastic process, where newly generated individuals are immediately dislocated at a different site. In our deterministic interpretation, it refers to the fact that if a cell divides, one daughter cell occupies the location of the mother cell while the other cell distributes to additional space, usually in direct proximity. For this analysis, cells are assumed to be very small compared to the size of the tissue domain $\Omega$ (which we take without loss of generality to have unit volume), and are small even compared to integration increments $d x$ and $d y$. Let $u(x, t)$ denote the density (in "cells per unit cell space," i.e., the fraction of the interval $(x, x+d x)$ physically occupied by cells) of cancer stem cells (CSCs) and $v(x, t)$ the density of nonstem cancer cells (TCs) at time $t$ and location $x$. We define the total tumor density 
$p(x, t)=u(x, t)+v(x, t)$. We also assume cells cannot pile on top of one another, so there is a maximum density of one cell per unit cell space. This implies $p(x, t) \leq 1$. Cells can only proliferate if there is space for the cell to place a daughter cell, and reproduction is inhibited otherwise (cellular quiescence). To model the spatial search for space, we define a nonlinear integral term. In line with the agent-based model (Enderling et al. 2009), we assume that all cells can migrate randomly, which we model by simple diffusion. These assumptions lead to the following coupled system of equations describing CSC and TC dynamics:

$$
\text { CSC: } \quad \begin{aligned}
\frac{\partial u(x, t)}{\partial t} & =D_{u} \Delta u+\delta \gamma \int_{\Omega} k(x, y, p(x, t)) u(y, t) d y \\
\mathrm{TC}: \quad \frac{\partial v(x, t)}{\partial t}= & D_{v} \Delta v+(1-\delta) \gamma \int_{\Omega} k(x, y, p(x, t)) u(y, t) d y \\
& -\alpha v+\rho \int_{\Omega} k(x, y, p(x, t)) v(y, t) d y .
\end{aligned}
$$

The spatial distribution kernel $k(x, y, p)$ describes the rate of progeny contribution to location $x$ from a cell at location $y$, per "cell cycle time" (i.e., the defined period between divisions of a freely-cycling cell). Of note, it follows that $k(x, y, p(x, t)) \leq$ 1 , since one cannot distribute more than one cell to $x$, per cell at $y$, per cell cycle time, and $\int_{\Omega} k(x, y, p(x, t)) d x$ is $\leq 1$, because this integral is equal to the total rate of progeny occupation over the domain, per parent cell at $y$, per cell cycle time, which of course can also be no more than 1 . Since greater density at $x$ would be expected to hinder progeny occupation, we further assume $k$ is monotonically decreasing in $p$ with $k(x, y, p(x, t))=0$ at $p=1$. The number of cell cycle times per unit time of CSCs and TCs are denoted by $\gamma>0$ and $\rho>0$, respectively. The parameter $\delta$ with $0 \leq \delta \leq 1$ denotes the fraction of CSC divisions that are symmetric, and $\alpha>0$ denotes the TC death rate. Background cell motility for CSCs and TCs is described by diffusion with coefficients $D_{u}>0, D_{v}>0$, respectively. The system is considered to hold in the spatial domain $\Omega=\mathbb{R}^{n}$, a smooth bounded domain with homogeneous Neumann or Dirichlet boundary conditions:

(bc1) Homogeneous Neumann boundary conditions correspond to a tissue surrounded by membranes, smooth muscle tissue, or bone, which, for the purpose of the model are impenetrable by cells. In that case, the boundary conditions are

$$
\frac{\partial}{\partial n} u=0, \quad \frac{\partial}{\partial n} v=0 \quad \text { on } \partial \Omega,
$$

where $\partial / \partial n$ denotes the normal derivative at the boundary. The redistribution kernel can only redistribute cells within this domain $\Omega$, hence we impose:

$$
k(x, y, p)=0 \quad \text { for all } x \notin \Omega .
$$

(bc2) Homogeneous Dirichlet boundary conditions correspond to tissues that cells can freely leave but not reenter again such as intravasation into adjacent blood vessels (this assumption can later be relaxed as to allow for tumor self-seeding by circulating cells (Kim et al. 2009)). Hence,

$$
u=0, \quad v=0 \quad \text { on } \partial \Omega .
$$


The redistribution kernel describes transport of cells out of the domain but does not allow entering from the outside if

$$
k(x, y, p)=0 \quad \text { for } y \notin \Omega .
$$

Based on the two boundary conditions we can model any combination of domains (such as partly covered by membranes, partially permeable membranes and adjacent blood vessels). Herein, however, for simplicity, we only demonstrate two basic cases.

\subsection{Model Reductions}

The system of integro-differential equations (1) and (2) is difficult to analyze analytically. We therefore introduce two simplifying assumptions to reduce the model to a system of two ODEs.

Reduction 1: Progeny Placement Depends Only on the Density at the Destination In this case:

$$
k=k(p(x, t)) .
$$

We introduce mean densities, which, if we now assume (without loss of generality) that the domain $\Omega$ has unit volume, can be written as

$$
\bar{u}(t):=\int_{\Omega} u(y, t) d y, \quad \bar{v}(t):=\int_{\Omega} v(y, t) d y, \quad \bar{p}(t)=\bar{u}(t)+\bar{v}(t) .
$$

Then (1) and (2) become

$$
\begin{aligned}
& u_{t}(x, t)=D_{u} \Delta u+\delta \gamma k(p(x, t)) \bar{u}(t), \\
& v_{t}(x, t)=D_{v} \Delta v+(1-\delta) \gamma k(p(x, t)) \bar{u}(t)-\alpha v(x, t)+\rho k(p(x, t)) \bar{v}(t),
\end{aligned}
$$

with appropriate boundary conditions as described above.

Reduction 2: Density is Uniform Across the Domain We next assume tumor growth to be uniform across the domain, in which case, $k(p(x, t))$ can be expressed as $k(\bar{p}(t)), u(x, t)$ and $v(x, t)$ can be replaced with their spatially-averaged values, and diffusion is zero everywhere. Equations (6) and (7) now become

$$
\begin{aligned}
\bar{u}_{t}(t) & =\delta \gamma k(\bar{p}(t)) \bar{u}(t), \\
\bar{v}_{t}(t) & =(1-\delta) \gamma k(\bar{p}(t)) \bar{u}(t)-\alpha \bar{v}(t)+\rho k(\bar{p}(t)) \bar{v}(t) .
\end{aligned}
$$

We assume

(A1) $k(\bar{p}(t))$ is piecewise differentiable, $k(\bar{p}(t))>0$ for $0 \leq \bar{p}(t)<1, k(\bar{p}(t))=0$ for $p(t) \geq 1$ and $k(\bar{p}(t))$ is decreasing for $0 \leq \bar{p}(t)<1$.

For the numerical simulations below, we will consider the standard example for $k$

$$
k(\xi)=\max \left\{1-\xi^{\sigma}, 0\right\}, \quad \sigma \geq 1 .
$$

An exponent of $\sigma=1$ corresponds to a linearly decreasing rate of occupancy for newborn cells as the target density $\bar{p}(t)$ increases. However, cells are nonrigid and 
Fig. 3 Comparison of two volume filling constraints $k(P)=\max \left\{1-P^{4}, 0\right\}$ (black, solid) and $1-P$ (blue, dotted) (Color figure online)

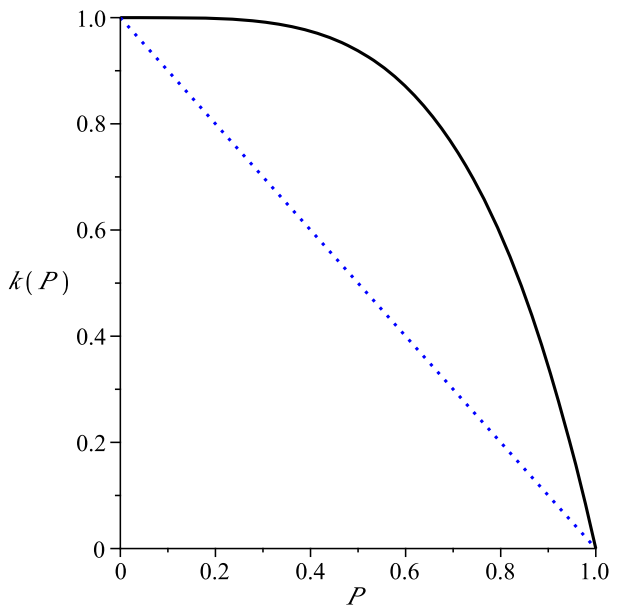

deformable and able to squeeze into available open spaces. Therefore, $k(\bar{p}(t))$ should be decreasing in $\bar{p}(t)$ but be greater than $1-\bar{p}(t)$ for $0<\bar{p}(t)<1$ (see Fig. 3 ). Comparable to a chemotaxis model (Wang and Hillen 2007) we choose $\sigma>1$ (e.g., $\sigma=4$ in Fig. 3).

For simplicity, we further assume the CSC and TC growth rates to be

(A2) $\gamma=\rho=1$.

Under assumption (A1), Eqs. (8) and (9) become:

$$
\begin{aligned}
\bar{u}_{t}(t) & =\delta k(\bar{p}(t)) \bar{u}(t), \\
\bar{v}_{t}(t) & =(1-\delta) k(\bar{p}(t)) \bar{u}(t)+k(\bar{p}(t)) \bar{v}(t)-\alpha \bar{v}(t) .
\end{aligned}
$$

We show the following main result for model $(11,12)$.

Theorem 2.2 Assume (A1). Then model $(11,12)$ shows the tumor growth paradox as defined in Definition 2.1.

This is now demonstrated, along with a full model analysis, in the next section.

\section{Analysis of the Reduced ODE System}

Some Basic Properties By adding Eqs. $(11,12)$, we obtain the behavior of the total tumor population density $\bar{p}(t)=\bar{u}(t)+\bar{v}(t)$ over time:

$$
\bar{p}_{t}(t)=k(\bar{p}(t)) \bar{p}(t)-\alpha \bar{v}(t) .
$$

The growth rate $\bar{p}_{t}(t) / \bar{p}(t)$ of the total population is given by $k(\bar{p}(t))$ and reduced by $-\alpha \bar{v}(t) / \bar{p}(t)$. In line with observations in the computational model (Enderling et al. 2009), a large $\bar{v}$-compartment (TC) decelerates overall tumor progression.

Without a CSC population density $\bar{u}(t)$, the population density of TC, $\bar{v}(t)$, satisfies the equation

$$
\bar{v}_{t}(t)=k(\bar{v}(t)) \bar{v}(t)-\alpha \bar{v}(t) .
$$


Since $k(\bar{v}(t))$ is a decreasing function of $\bar{v}(t)$ per (A1), the differentiated cell population dies out when

$$
\alpha>k(0) \text {. }
$$

Since there is no proliferation for $\bar{u}(t)+\bar{v}(t)>1$, we can restrict analysis to the triangular domain:

$$
\Delta:=\{(\bar{u}(t), \bar{v}(t)): 0 \leq \bar{u}(t) \leq 1, \bar{v}(t) \geq 0, \bar{u}(t)+\bar{v}(t) \leq 1\} .
$$

From $(11,12)$, it is obvious that $\Delta$ is positively invariant. Note that along the line $\bar{u}(t)+\bar{v}(t)=1$ the vector field is given by $(0,-\alpha \bar{v}(t))^{T}$, which points into $\Delta$.

\subsection{Steady States}

The steady states of system $(11,12)$ are given by

$$
X_{0}=(0,0), \quad X_{v}=\left(0, v_{0}\right) \quad \text { and } \quad X_{u}=(1,0),
$$

where $v_{0}$ solves $k\left(v_{0}\right)=\alpha$. The Jacobian of the vector field $f(\bar{u}(t), \bar{v}(t))$ is given by

$$
D f(\bar{u}, \bar{v})=\left(\begin{array}{cc}
\delta k^{\prime} \bar{u}+\delta k & \delta k^{\prime} \bar{u} \\
(1-\delta)\left(k^{\prime} \bar{u}+k\right)+k^{\prime} \bar{v} & (1-\delta) k^{\prime} \bar{u}+k^{\prime} \bar{v}+k-\alpha
\end{array}\right) .
$$

The eigenvalues of $D f(0,0)$ are $\lambda_{1}=\delta k(0)>0$ and $\lambda_{2}=k(0)-\alpha$. Hence, $X_{0}=(0,0)$ is a saddle point for $\alpha>k(0)$ and an unstable node for $\alpha<k(0)$. The eigenvectors corresponding to $\lambda_{1}$ and $\lambda_{2}$ are $\zeta_{1}=(\alpha-(1-\delta) k(0),(1-\delta) k(0))^{T}$ and $\zeta_{2}=(0,1)^{T}$, respectively. If $\alpha>(1-\delta) k(0)$, the eigenvector $\zeta_{1}$ has two positive entries and a decreasing slope in $\alpha$ (a fact that will become important later).

The eigenvalues of $D f\left(0, v_{0}\right)$ are $\lambda_{1}=\alpha \delta>0$ and $\lambda_{2}=k^{\prime}\left(v_{0}\right) v_{0}<0$ with corresponding eigenvectors

$$
\zeta_{1}=\left(\begin{array}{c}
\delta \alpha-k^{\prime}\left(v_{0}\right) v_{0} \\
(1-\delta) \alpha+k^{\prime}\left(v_{0}\right) v_{0}
\end{array}\right), \quad \zeta_{2}=\left(\begin{array}{l}
0 \\
1
\end{array}\right) .
$$

For the steady states of the form $X_{U}=(1,0)$, the linearization becomes

$$
D f(1,0)=\left(\begin{array}{cc}
-\delta \kappa & -\delta \kappa \\
-(1-\delta) \kappa & -(1-\delta) \kappa-\alpha
\end{array}\right),
$$

where $\kappa=-k^{\prime}(1)$ denotes a positive constant. We find a trace of $-\kappa-\alpha$ and a positive determinant of $\delta \kappa \alpha$. Hence, $(1,0)$ is a stable node or stable spiral. Consequently, $(1,0)$ is the only attractor in the positively invariant region $\Delta$ and there are no other fixed points inside $\Delta$. By the Poincare-Bendixson theorem, we obtain the following lemma.

Lemma 3.1 The steady state $(1,0)$ of $(11,12)$ is globally asymptotically stable in the simplex region $\Delta$.

We have shown that the pure stem cell state $(1,0)$ is a global attractor. If time goes to infinity, the tumor will consist of stem cells only. In Fig. 4, we present a corresponding long-time simulation of the individual based model (Enderling et al. 2009). We see that in a spatially constrained domain the stem cell compartment and 

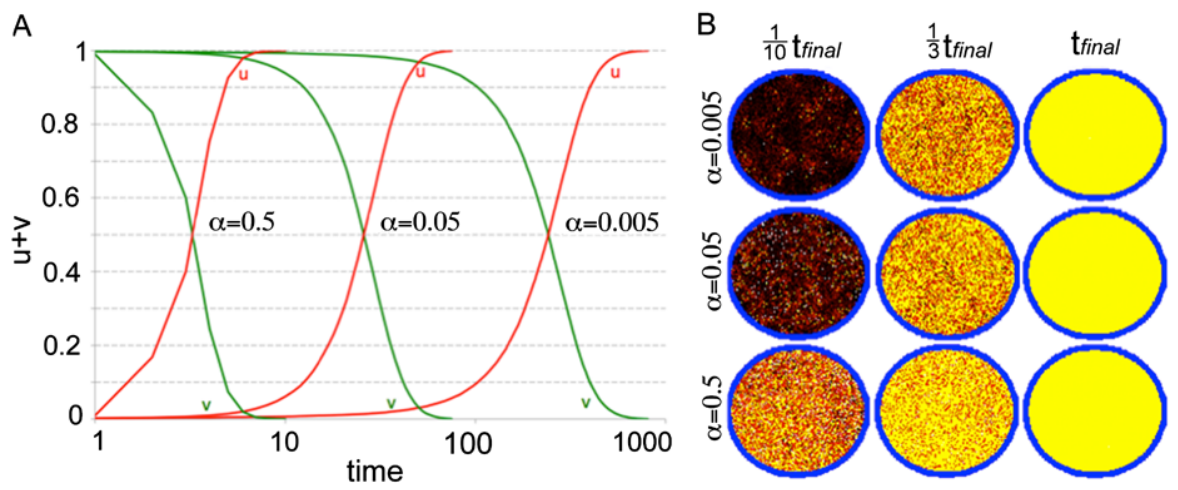

Fig. 4 Long time simulation of the agent-based model in a small circular domain. For all three choices of death rate $\alpha$, we observe convergence to the pure stem-cell state. Note that here are no nutrient restrictions

fraction increases with time and eventually becomes a pure population. Intermediate tumor consistency and steady state time are dependent on cell death rate $\alpha$. The convergence to $(1,0)$ does not interfere with our analysis, since we are not interested in the long time dynamics $(t \rightarrow \infty)$, but rather in the intermediate time dynamics of the tumor. We use numerical simulations and geometric singular perturbation theory to explore this further.

\subsection{Numerical Examples}

For numerical simulations of model $(11,12)$, we choose parameter values of

$$
k(\bar{p}(t))=1-\bar{p}^{4}(t), \quad \delta=0.01
$$

and $\alpha$ is varied as bifurcation parameter. We chose an exponent of $p^{4}$ to obtain a function $k$ which lies well above the linear curve $1-p$ (see Fig. 3). We have tested many more exponents and functional forms for $k$ and it turns out that the tumor growth paradox is more pronounced for functions that are well above $1-p$. The above choice forms a good representation of our results, however, the paradox is true for each decreasing function $k$ which satisfies the basic assumptions in (A1).

We use Maple to visualize the phase portraits. Figure 5A shows five typical trajectories for a cell death rate of $\alpha=1$. Solutions start close to the $\bar{u}$ axis and grow quickly in $\bar{v}$, but then settle on a common orbit slowly increasing in $\bar{u}$ and decreasing in $\bar{v}$. We will show later that this dynamic can be analyzed by geometric singular perturbation theory (Sect. 3.3) (Jones 1994). Hek recently reviewed the geometric singular perturbation theory and the application of the Fenichel theorems to biological modeling (Hek 2010). We observe a fast convergence towards a slow manifold describing the "common orbit" (Fig. 5A). To compare solutions for different values of $\alpha$ we visualize their trajectories together in phase portrait (Fig. 5B) . The behavior for all choices of $\alpha$ are comparable for a total time of $t=300$. However, the $\bar{v}$-component is significantly 

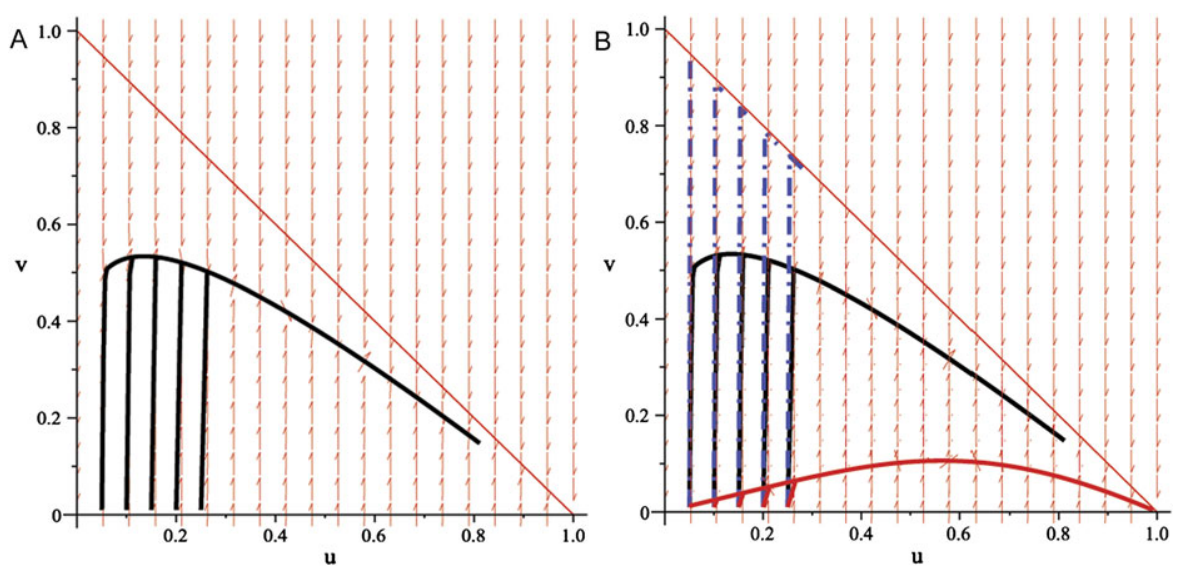

Fig. 5 (A) Five typical trajectories for $\alpha=1$ and other parameters from (15). The diagonal line indicates the invariant domain $\Delta$. (B) Typical trajectories for $\alpha=0.05$ (blue, dashdot), 1 (black, solid), 5 (red dash) and other parameters from (15). All orbits are run for $T=300$ time units (Color figure online)

larger for smaller $\alpha$ values $(\alpha=0.05)$, and the trajectories for the largest investigated cell death rate $(\alpha=5)$ rapidly approach a pure CSC state at the attractor $(1,0)$.

The time evolution of the total population density $\bar{p}(t)$ from a common initial condition of $(u(0)=0.05, v(0)=0.01)$ for $\alpha=0.05,1,5$ is shown in Fig. 6 . With increasing cell death rate the overall tumor population density initially grows slower, but eventually exceeds those with lower death rates. The positive correlation of cell death and tumor growth becomes apparent after 650 time units with $\bar{p}(t)_{\alpha=5}>\bar{p}(t)_{\alpha=1}>\bar{p}_{\alpha=0.05}$. These results confirm the observations of the tumor growth paradox in the above discussed agent-based model (Enderling et al. 2009). Other values for cell death rate parameter $\alpha$ and different interaction kernel $k$ further confirm this, and the paradox is enhanced for more restrictive kernels, for example, for $\sigma=1$ or $k(\bar{p}(t))=k_{2}(\bar{p}(t))=$ $\left\{e^{-5 \bar{p}(t)}-e^{-5}\right\}$ (simulations not shown). Interestingly, the evolution of the CSC and TC ratio over time differs for different values of $\alpha$ (Fig. 7). For a small death rate $(\alpha=0.05)$, the population is rapidly dominated by TC. However, within the same time, interval CSC becomes dominant and even exclusive for larger $\alpha$ values, which explains the increasing tumor size for increasing cell death rates $\alpha$.

\subsection{Geometric Singular Perturbation Analysis}

We use a geometric singular perturbation approach (Jones 1994; Hek 2010) to prove that the solutions rapidly approach the slow manifold, which is the outer solution of system $(11,12)$. On the slow manifold, we identify the tumor growth paradox as a typical and robust property of this model (Theorem 3.3). The small parameter in the analysis is the rate of symmetric CSC division $\delta$ (this is a CSC gives rise to two CSC). 

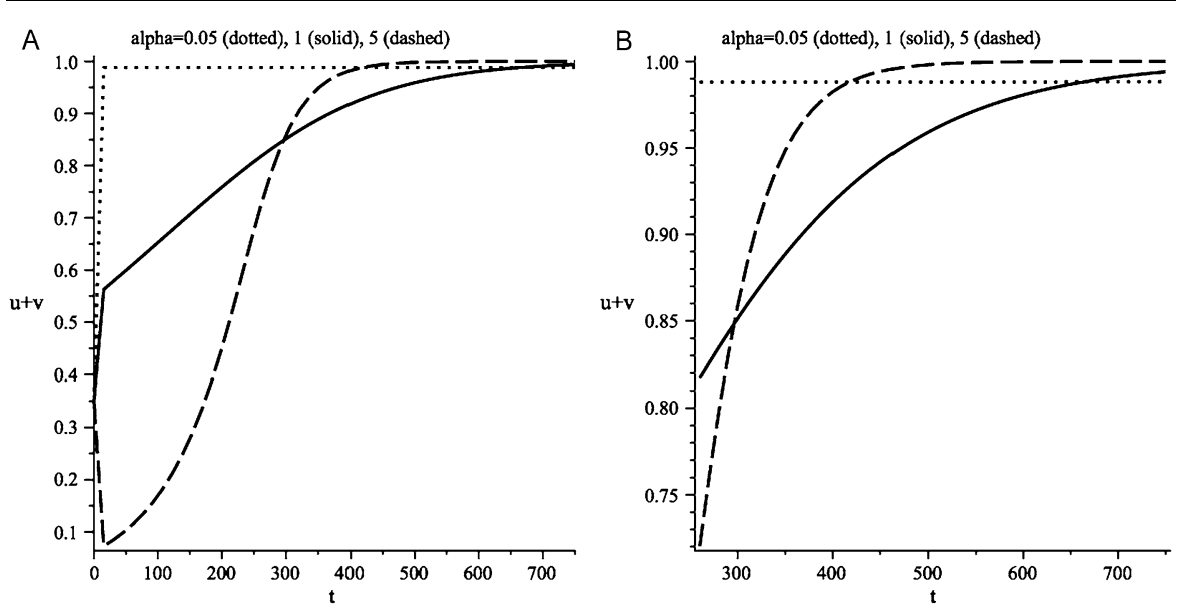

Fig. 6 Time evolution of the total tumor population for $\alpha=0.05$ (dotted), $\alpha=1$ (solid), $\alpha=5$ (dashed). The initial condition for all orbits is $(0.05,0.3)$ and other parameters are as per (15). (A) Time range of $[0,750],(\mathbf{B})$ time range of $[250,750]$ to highlight that orbits change order
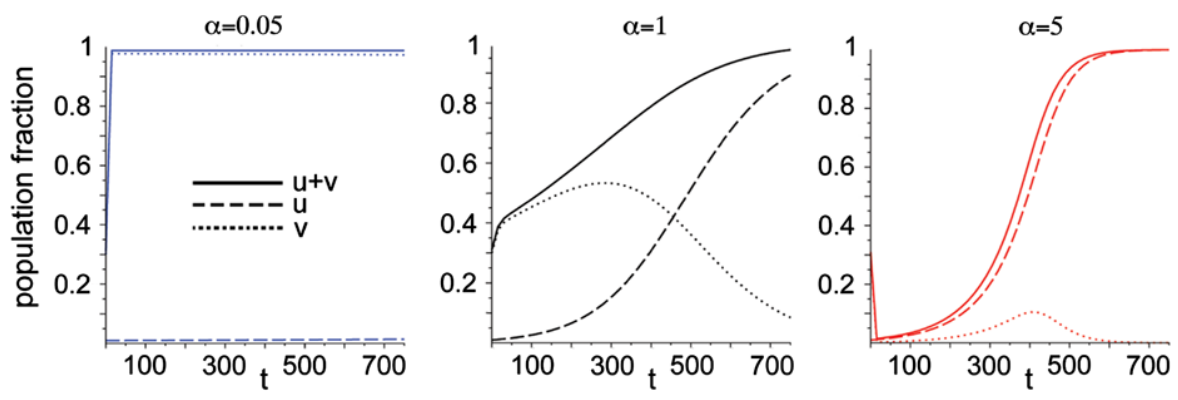

Fig. 7 Time evolution of the cancer stem cell population $u$ (dashed), the nonstem cancer cell population $v$ (dotted) and the total population $u+v$ (solid) for different $\alpha$ values

\subsubsection{Inner Solution}

System $(11,12)$ with $\delta \ll 1$ denotes the fast system. Solutions of the fast system are the inner solutions and to leading order, the inner solution $\left(\bar{u}_{\mathrm{i} n}(t), \bar{v}_{\mathrm{i} n}(t)\right)$ satisfies

$$
\begin{aligned}
\bar{u}_{t}(t) & =0, \\
\bar{v}_{t}(t) & =k(\bar{p}(t)) \bar{u}(t)+k(\bar{p}(t)) \bar{v}(t)-\alpha \bar{v}(t) .
\end{aligned}
$$

Hence, $\bar{u}_{\text {in }}(t)$ is constant and $\bar{v}_{\text {in }}(t)$ solves the differential equation (16).

The steady state of this equation is given by

$$
(\alpha-k(\bar{p}(t))) \bar{v}(t)=k(\bar{p}(t)) \bar{u}(t),
$$

which describes the slow manifold of the system:

$$
M:=\left\{\left(\bar{u}_{M}, \bar{v}_{M}\right): k\left(\bar{p}_{M}\right) \bar{p}_{M}=\alpha \bar{v}_{M}, \bar{p}_{M}=\bar{u}_{M}+\bar{v}_{M}\right\} .
$$


We show that this manifold is normally hyperbolic, meaning in this case that it is attractive for the fast dynamics or inner solution. Demonstrating this, we linearize (16) with respect to a $\bar{v}$-perturbation from a point $\left(\bar{u}_{M}, \bar{v}_{M}\right) \in M$ using $(\bar{u}(t), \bar{v}(t))=$ $\left(\bar{u}_{M}, \bar{v}_{M}+\tilde{v}(t)\right)$. The small perturbation $\tilde{v}(t)$ satisfies.

$$
\frac{d}{d t} \tilde{v}(t)=\left(k\left(\bar{p}_{M}\right)-\alpha+k^{\prime}\left(\bar{p}_{M}\right)\left(\bar{u}_{M}+\bar{v}_{M}\right)\right) \tilde{v}(t) .
$$

Noting from the definition of $M$ that $\left(\alpha-k\left(\bar{p}_{M}\right)\right)=k\left(\bar{p}_{M}\right) \bar{u}_{M} / \bar{v}_{M}$, the above equation can be rewritten as

$$
\frac{d}{d t} \tilde{v}(t)=\left(-k\left(\bar{p}_{M}\right) \bar{u}_{M} / \bar{v}_{M}+k^{\prime}\left(\bar{p}_{M}\right)\left(\bar{u}_{M}+\bar{v}_{M}\right)\right) \tilde{v}(t) .
$$

Since $k$ is decreasing in the domain $\Delta$, we find that the leading coefficient on the right is always negative. Hence, the slow manifold is normally hyperbolic (see Jones 1994; Hek 2010). Furthermore, Fenichel's theorems guarantee that for $\delta$ small enough there exists an invariant manifold $M_{\delta}$ of the whole system $(11,12)$, which is close to $M$, and $M_{\delta}$ can be written as graph on $M$.

\subsubsection{Outer Solution}

The long time dynamics is given by the outer solution on $M$. To obtain the outer solution, we rescale the original system $(11,12)$ by a slow time scale $\tau=\delta t$. We obtain

$$
\begin{aligned}
\bar{u}_{\tau}(\tau) & =k(\bar{p}(\tau)) \bar{u}(\tau), \\
\delta \bar{v}_{\tau}(\tau) & =(1-\delta) k(\bar{p}(\tau)) \bar{u}(\tau)+k(\bar{p}(\tau)) \bar{v}(\tau)-\alpha \bar{v}(\tau) .
\end{aligned}
$$

The leading order terms provide the outer solution $\left(\bar{u}_{\text {out }}(\tau), \bar{v}_{\text {out }}(\tau)\right)$, defined by

$$
\begin{aligned}
\frac{d}{d \tau} \bar{u}_{\text {out }}(\tau) & =k\left(\bar{p}_{\text {out }}(\tau)\right) \bar{u}_{\text {out }}(\tau), \\
0 & =k\left(\bar{p}_{\text {out }}(\tau)\right) \bar{u}_{\text {out }}(\tau)+k\left(\bar{p}_{\text {out }}(\tau)\right) \bar{v}_{\text {out }}(\tau)-\alpha \bar{v}_{\text {out }}(\tau) .
\end{aligned}
$$

The second equation is solved on the slow manifold $M$ as given above, and the dynamics on $M$ is given by the first equation of (19). We study $M$ in more detail.

Lemma 3.2 The slow manifold can be written as a graph $(\bar{u}, \bar{v})=\left(\bar{u}, v_{M}(\bar{u})\right)$. Furthermore,

$$
\frac{d}{d \bar{u}} v_{M}(\bar{u})=\frac{k^{\prime}(\bar{p}) \bar{p}+k(\bar{p})}{\alpha-k^{\prime}(\bar{p}) \bar{p}-k(\bar{p})}, \quad \text { with } \bar{p}=\bar{u}+v_{M}(\bar{u}) .
$$

Given two death rates $\alpha_{1}>\alpha_{2}$ then the slow manifold for $\alpha_{1}$ is below the slow manifold of $\alpha_{2}$, i.e.

$$
v_{M}^{(1)}(\bar{u})<v_{M}^{(2)}(\bar{u})
$$

Proof The slow manifold can be written as

$$
(\alpha-k(\bar{u}+\bar{v})) \bar{v}=k(\bar{u}+\bar{v}) \bar{u} .
$$


If we fix $0<\bar{u}<1$, then the left hand side is increasing in $\bar{v}$ and the right-hand side is decreasing. Further, for $\bar{v} \rightarrow 0$, the left-hand side approaches 0 and the right-hand side approaches $k(\bar{u}) \bar{u}>0$. For $\bar{v} \rightarrow 1$, the left-hand side approaches $\alpha>0$ and the right-hand side approaches 0 . Hence, for each $0<\bar{u}<1$, there is exactly one solution $\bar{v}=v_{M}(\bar{u})$ of (21). Hence, for $0<\bar{u}<1$, the slow manifold can be written as a graph $v_{M}(\bar{u})$ :

$$
k\left(\bar{u}+v_{M}(\bar{u})\right)\left(\bar{u}+v_{M}(\bar{u})\right)=\alpha v_{M}(\bar{u}) .
$$

We differentiate this equation with respect to $\bar{u}$, where we omit the arguments in the functions for transparency (i.e., $k^{\prime}$ stands for $k^{\prime}\left(\bar{u}+v_{M}(\bar{u})\right)$, etc.):

$$
\begin{aligned}
k^{\prime}\left(1+v_{M}^{\prime}\right)\left(\bar{u}+v_{M}\right)+k\left(1+v_{M}^{\prime}\right) & =\alpha v_{M}^{\prime}, \\
k^{\prime}\left(\bar{u}+v_{m}\right)+k & =v_{M}^{\prime}\left(\alpha-k^{\prime}\left(\bar{u}+v_{M}\right)-k\right), \\
v_{M}^{\prime} & =\frac{k^{\prime}\left(\bar{u}+v_{M}\right)+k}{\alpha-k^{\prime}\left(\bar{u}+v_{M}\right)-k},
\end{aligned}
$$

which proves the formula (20) for $0<\bar{u}<1$.

Next, we need to study the limits of $\bar{u} \rightarrow 0,1$. The $\bar{v}$-axis intercept of the slow manifold is given for $\bar{u}=0$ and

$$
(\alpha-k(\bar{v})) \bar{v}=0
$$

hence either $\bar{v}=0$ or $k(\bar{v})=\alpha$. If the kernel $k$ is chosen such that $k(0)<\alpha$, then the second condition cannot be satisfied and the slow manifold connects to $(0,0)$. If $0<\alpha<k(0)$, then there is a unique $\bar{v}^{*}>0$ such that $k\left(\bar{v}^{*}\right)=\alpha$, and indeed $\left(0, \bar{v}^{*}\right)$ is a steady state.

We evaluate the above derivative at the point $\left(0, \bar{v}^{*}\right)$ and using the fact that $k\left(\bar{v}^{*}\right)=$ $\alpha$, we find

$$
\frac{d}{d \bar{u}} v_{M}(0)=-\frac{\alpha+k^{\prime}\left(\bar{v}^{*}\right) \bar{v}^{*}}{k^{\prime}\left(\bar{v}^{*}\right) \bar{v}^{*}}
$$

This, in fact, is identical to the slope of the unstable eigenvector of $\left(0, \bar{v}^{*}\right)$ as found earlier in (14) for $\delta \rightarrow 0$. Using the implicit function theorem, the slow manifold, starting at $\left(0, \bar{v}^{*}\right)$ can be locally continued in direction of the unstable eigenvector for $\bar{u}>0$. Hence, the slow manifold indeed connects to this point $\left(0, \bar{v}^{*}\right)$. For convenience, we denote $\bar{v}^{*}=0$ for the case of $k(0)<\alpha$.

For $\bar{u} \rightarrow 1$, we find $\lim _{\bar{u} \rightarrow 1} v_{M}(\bar{u})=0$. Moreover, the above formula (20) is continuous for $\bar{u} \rightarrow 1$. Hence, the slow manifold can be written as a differentiable graph for all $0 \leq \bar{u} \leq 1$.

Finally, for fixed $\bar{u}$, the left-hand side of (21) is increasing in $\alpha$, hence the point $v_{M}(\bar{u})$ is decreasing in $\alpha$.

Figure 8 shows three slow manifolds for the choices of $\alpha=0.05,1.0,5.0$. We see that for increasing $\alpha$ value, the slow manifold is lower. 
Fig. 8 The slow manifolds for $\alpha=0.05$ (blue, dashdot), $\alpha=1$ (black, solid), $\alpha=5$ (red, dash) (Color figure online)

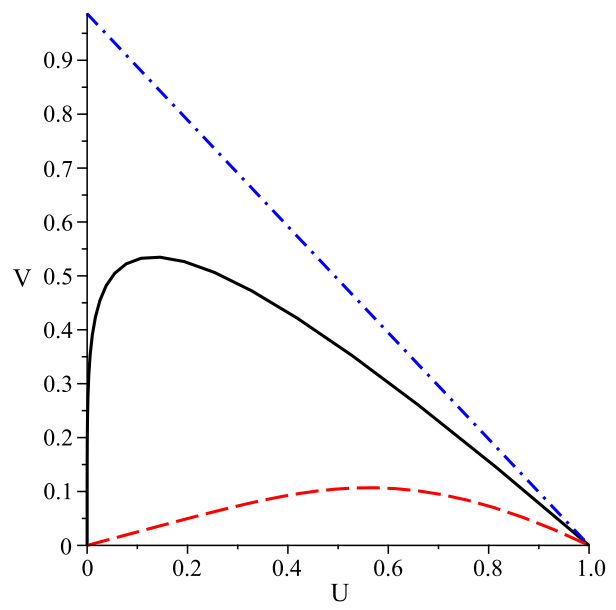

\subsubsection{Tumor Growth Paradox}

Now we are ready to formulate the tumor growth paradox. There are several ways how the paradox can arise. We might want to focus on tumors that are initially of the same size, or tumors that have comparable stem cell numbers. We will first formulate the general result and then discuss the various implications.

Theorem 3.3 (Tumor growth paradox) Assume $\alpha_{1}>\alpha_{2}>0$ and let $\bar{p}_{1}(t)=\bar{u}_{1}(t)+$ $\bar{v}_{1}(t)$ and $\bar{p}_{2}(t)=\bar{u}_{2}(t)+\bar{v}_{2}(t)$ denote the corresponding solutions of the stem cell model $(11,12)$. We assume that the tumor dynamics has settled onto the slow manifold $M$ and that at time $t_{0} \geq 0$ two tumors of equal size are presented $\bar{p}_{1}\left(t_{0}\right)=\bar{p}_{2}\left(t_{0}\right)=\tilde{p}$, with $0<\tilde{p}<1$. Then

$$
\frac{d}{d t} \bar{p}_{1}\left(t_{0}\right)>\frac{d}{d t} \bar{p}_{2}\left(t_{0}\right)
$$

and

$$
\bar{p}_{1}(t)>\bar{p}_{2}(t), \quad \text { for all } t>t_{0} .
$$

Proof We have seen earlier that the slow manifold can be written as a graph $\left(\bar{u}, v_{M}(\bar{u})\right)$. Hence, the growth rate of a tumor on $M$ can be written as

$$
\frac{d}{d t} \bar{p}(t)=\frac{d}{d t} \bar{u}(t)+v_{M}^{\prime}(\bar{u}(t)) \frac{d}{d t} \bar{u}(t)=\frac{d}{d t} \bar{u}(t)\left(1+v_{M}^{\prime}(\bar{u}(t))\right) .
$$

From Lemma 3.2, we find

$$
v_{M}^{\prime}=\frac{k^{\prime}\left(\bar{u}+v_{M}\right)+k}{\alpha-k^{\prime}\left(\bar{u}+v_{M}\right)-k} .
$$

Then from (22), we obtain 


$$
\begin{aligned}
\frac{d}{d t} \bar{p}(t) & =k \bar{u}\left(1+\frac{k^{\prime}\left(\bar{u}+v_{M}\right)+k}{\alpha-k^{\prime}\left(\bar{u}+v_{M}\right)-k}\right) \\
& =k \bar{u}\left(\frac{\alpha-k^{\prime}\left(\bar{u}+v_{M}\right)-k+k^{\prime}\left(\bar{u}+v_{M}\right)+k}{\alpha-k^{\prime}\left(\bar{u}+v_{M}\right)-k}\right) \\
& =\frac{\alpha k \bar{u}}{\alpha-k^{\prime}\left(\bar{u}+v_{M}\right)-k} .
\end{aligned}
$$

Written out with complete dependencies, the last formula reads

$$
\frac{d}{d t} \bar{p}(t)=\frac{\alpha k(\bar{p}(t)) \bar{u}(t)}{\alpha-k^{\prime}(\bar{p}(t)) \bar{p}(t)-k(\bar{p}(t))} .
$$

Now we come back to the two tumors $\bar{p}_{1}(t), \bar{p}_{2}(t)$ with equal size at time $t_{0}, \tilde{p}=$ $\bar{p}_{1}\left(t_{0}\right)=\bar{p}_{2}\left(t_{0}\right)$. The growth rates for $i=1,2$ at time $t_{0}$ are

$$
\frac{d}{d t} \bar{p}_{i}\left(t_{0}\right)=\frac{\alpha_{i} k(\tilde{p}) \bar{u}_{i}\left(t_{0}\right)}{\alpha_{i}-k^{\prime}(\tilde{p}) \tilde{p}-k(\tilde{p})} .
$$

Next, we replace the term $\bar{u}_{i}\left(t_{0}\right)$ in this formula. On $M$, we have a time $t_{0}$ that

$$
k(\tilde{p}) \tilde{p}=\alpha_{i} \bar{v}_{i}\left(t_{0}\right) .
$$

Hence,

$$
\alpha_{1} \bar{v}_{1}\left(t_{0}\right)=\alpha_{2} \bar{v}_{2}\left(t_{0}\right)
$$

and since $\alpha_{1}>\alpha_{2}$ it follows that $\bar{v}_{1}\left(t_{0}\right)<\bar{v}_{2}\left(t_{0}\right)$ and $\bar{u}_{1}\left(t_{0}\right)>\bar{u}_{2}\left(t_{0}\right)$. Moreover, for $i=1,2$,

$$
\bar{v}_{i}\left(t_{0}\right)=\frac{k(\tilde{p}) \tilde{p}}{\alpha_{i}}
$$

and

$$
\bar{u}_{i}\left(t_{0}\right)=\tilde{p}-\frac{k(\tilde{p}) \tilde{p}}{\alpha_{i}}=\frac{\left(\alpha_{i}-k(\tilde{p})\right) \tilde{p}}{\alpha_{i}} .
$$

Using this formula for $\bar{u}_{i}\left(t_{0}\right)$, we find from (24) that

$$
\frac{d}{d t} \bar{p}_{1}\left(t_{0}\right)>\frac{d}{d t} \bar{p}_{2}\left(t_{0}\right)
$$

is equivalent with

$$
\frac{k(\tilde{p})\left(\alpha_{1}-k(\tilde{p})\right) \tilde{p}}{\alpha_{1}-k^{\prime}(\tilde{p}) \tilde{p}-k(\tilde{p})}>\frac{k(\tilde{p})\left(\alpha_{2}-k(\tilde{p})\right) \tilde{p}}{\alpha_{2}-k^{\prime}(\tilde{p}) \tilde{p}-k(\tilde{p})} .
$$

Since $k(\tilde{p}) \tilde{p}>0$, the above inequality is equivalent with

$$
\frac{\alpha_{1}-k(\tilde{p})}{\alpha_{1}-k^{\prime}(\tilde{p}) \tilde{p}-k(\tilde{p})}>\frac{\alpha_{2}-k(\tilde{p})}{\alpha_{2}-k^{\prime}(\tilde{p}) \tilde{p}-k(\tilde{p})} .
$$

This last inequality is true, since the function

$$
f(\alpha):=\frac{\alpha-k(\tilde{p})}{\alpha-k^{\prime}(\tilde{p}) \tilde{p}-k(\tilde{p})}
$$


is monotonically increasing in $\alpha$; indeed

$$
f^{\prime}(\alpha)=\frac{-k^{\prime}(\tilde{p}) \tilde{p}}{\left(\alpha_{1}-k^{\prime}(\tilde{p}) \tilde{p}-k(\tilde{p})\right)^{2}}>0,
$$

since $k^{\prime}<0$. Hence, $(25)$ is true.

Consequently, $\bar{p}_{1}(t)>\bar{p}_{2}(t)$ for a small time interval $t \in\left(t_{0}, t_{0}+\varepsilon\right)$. If for some later time $t_{00}$, we have $\bar{p}_{1}\left(t_{00}\right)=\bar{p}_{2}\left(t_{00}\right)$ then a similar argument as used above leads to a contradiction. Hence, we must have $\bar{p}_{1}(t)>\bar{p}_{2}(t)$ for all $t>t_{0}$.

We now compare two small tumors of equal size that start with one or a few CSCs, $\left(\bar{u}_{0}, \bar{v}_{0}\right)=\left(\bar{u}_{0}, 0\right)$ and have the same growth parameters, differing only in their values for $\alpha$, the rates of cell death. To do this, we run the solutions through the fast system and into the slow manifold, where they assume the points $\left(\bar{u}_{0}, \bar{v}_{10}\right),\left(\bar{u}_{0}, \bar{v}_{20}\right)$, respectively, where $\bar{v}_{10}<\bar{v}_{20}$. We next advance only the smaller tumor forward along the slow manifold until it is the same size as the larger. Then we are in the situation of the above Theorem 3.3 and we can compare their instantaneous growth rates to see the tumor growth paradox.

Corollary 3.4 Assume (A1) and consider two death rates satisfying $\alpha_{1}>\alpha_{2}>0$. Let $\bar{p}_{1}(t), \bar{p}_{2}(t)$ denote the corresponding solutions of $(11,12)$ with common initial condition $(\bar{u}(0), \bar{v}(0))$. Then there exist times $t_{1}, t_{2}$ such that

$$
\bar{p}_{1}\left(t_{1}\right)=\bar{p}_{2}\left(t_{2}\right) \quad \text { and } \quad \bar{p}_{1}\left(t_{1}+\theta\right)>\bar{p}_{2}\left(t_{2}+\theta\right) \quad \text { for all } \theta>0 .
$$

Thus, a tumor growth paradox always exists under these conditions.

Our results confirm the numerical findings above and generalize these findings for a wide class of models solely dependent on assumption (A1). We conclude that the defined tumor growth paradox - proportional acceleration of tumor growth with increasing cell death-is a typical phenomenon. Cell death is an intrinsic property of nonstem cancer cells within the cancer stem cell hypothesis. Cell death occurs due to several reasons, including exhaustion of proliferation potential, reduction of telomere ends, genomic instability, or external agents such as external stress or the immune system. In the next section, we will show that the immune system accelerates tumor evolution towards a pure cancer stem cell state by selective killing of nonstem cancer cells.

\section{Immune-Mediated Selection for Cancer Stem Cells}

The interaction of the immune system with cancer cells and the growing tumor has long been appreciated and it is subject of numerous mathematical models (DeLisi and Rescigno 1977; Kuznetsov 1987; Sherratt and Nowak 1992; Kirschner and Panetta 1998; de Pillis and Radunskaya 2001; Matzavinos and Chaplain 2004; D’Onofrio 2005; Bellomo and Delitala 2008). In most models (e.g., Mallet and De Pillis 2006; Liu et al. 2007), the immune response is modeled to be harmful to the cancer cells using law of mass action terms, which yields gross reduction in the 
tumor population. However, the immune system can be tumor-inhibiting as well as tumor-promoting (Prehn 1972; Dunn et al. 2004; Hanahan and Weinberg 2011; DeLisi and Rescigno 1977). In fact, here we show a case where the harmful influence of the immune system leads to CSC selection and as a result to a larger tumor. Hence, the immune system appears to be tumor promoting. The details of immune-mediated tumor progression are complicated and subject of ongoing research. Herein, we use a very simple model for tumor-induced immune response and subsequent cancer cell (TC) cytotoxicity. Immune cells are often stimulated through endothelial growth factors. We assume that the size of the tumor population is proportional to the production of these growth factors, hence we understand the tumor growth rate $\bar{p}_{t}=\bar{u}_{t}+\bar{v}_{t}$ as a driver of increased immune response. In turn, immune cells increase the TC death rate $\alpha$ before facing degradation with rate $\tau$. CSC are intrinsically more resistant and evade immune responses (Schatton and Frank 2009). For demonstration purposes, we assume CSC to be completely insensitive to immune-induced killing, which leads to the following system of equations as revisions of $(8,9)$ :

$$
\begin{aligned}
\bar{u}_{t} & =\delta \gamma k(\bar{p}) \bar{u}, \\
\bar{v}_{t} & =(1-\delta) \gamma k(\bar{p}) \bar{v}+\rho k(\bar{p}) \bar{v}-\alpha(\bar{w}) \bar{v}, \\
\bar{w}_{t} & =g\left(\bar{u}_{t}, \bar{v}_{t}\right)-\tau \bar{w},
\end{aligned}
$$

where the functional dependence $\alpha(\bar{w})$ describes the effect of the immune system on the cancer cells (TC) and the function $g\left(\bar{u}_{t}, \bar{v}_{t}\right)$ describes the activation of the immune system by the growing tumor. Here, we choose $g\left(\bar{u}_{t}, \bar{v}_{t}\right):=\kappa\left(\bar{u}_{t}+\bar{v}_{t}\right)$, where $\kappa \geq 0$ is a constant.

Figure 9 shows a representative numerical solution of the model. In those simulations, we choose $k(\bar{p})=\max \left(1-\bar{p}^{4}, 0\right), \delta=0.01, \gamma=1, \tau=0.001, \kappa=10$ and $\alpha(\bar{w})=0.5+0.5 \bar{w}$. On the top left of Fig. 9, we show the tumor growth without an immune response $(\kappa=0)$. The TC quickly become the dominant population, but later decrease as the CSC population expands. The immune response (not shown) is not stimulated in this case. If the stimulation of an immune response is turned on $(\kappa=10)$, then the tumor dynamics is quite different. On the top right of Fig. 9, we see that the immune response successfully decreases the total tumor population, which in turn allows CSC to expand more quickly. The CSC population is able to reach a dominating fraction of the tumor population. As seen on the bottom right of Fig. 9, the immune response is stimulated very quickly as it responds to the growing tumor and selects for CSC. This effect of the immune system is typical for all models of the type (26) which we studied. Combined with the intrinsic tumor progression kinetics, we can derive several conclusions and hypothesis:

1. The ratio of CSC in a solid tumor may not be constant but continuously evolve with time. With the presented model, it monotonically increases with time toward a pure CSC state. Hence, the ratio of stem cells in a tumor depends on its age.

2. The eventual dominance of CSC is accelerated by external agents that act to selectively kill non-stem cancer cells.

3. With CSC intrinsically evading immune responses (Schatton and Frank 2009), tumors grown in vivo are immuno-modulated, and thus the emerging population is selected for and enriched in CSC. If such a tumor is harvested and cultured in 

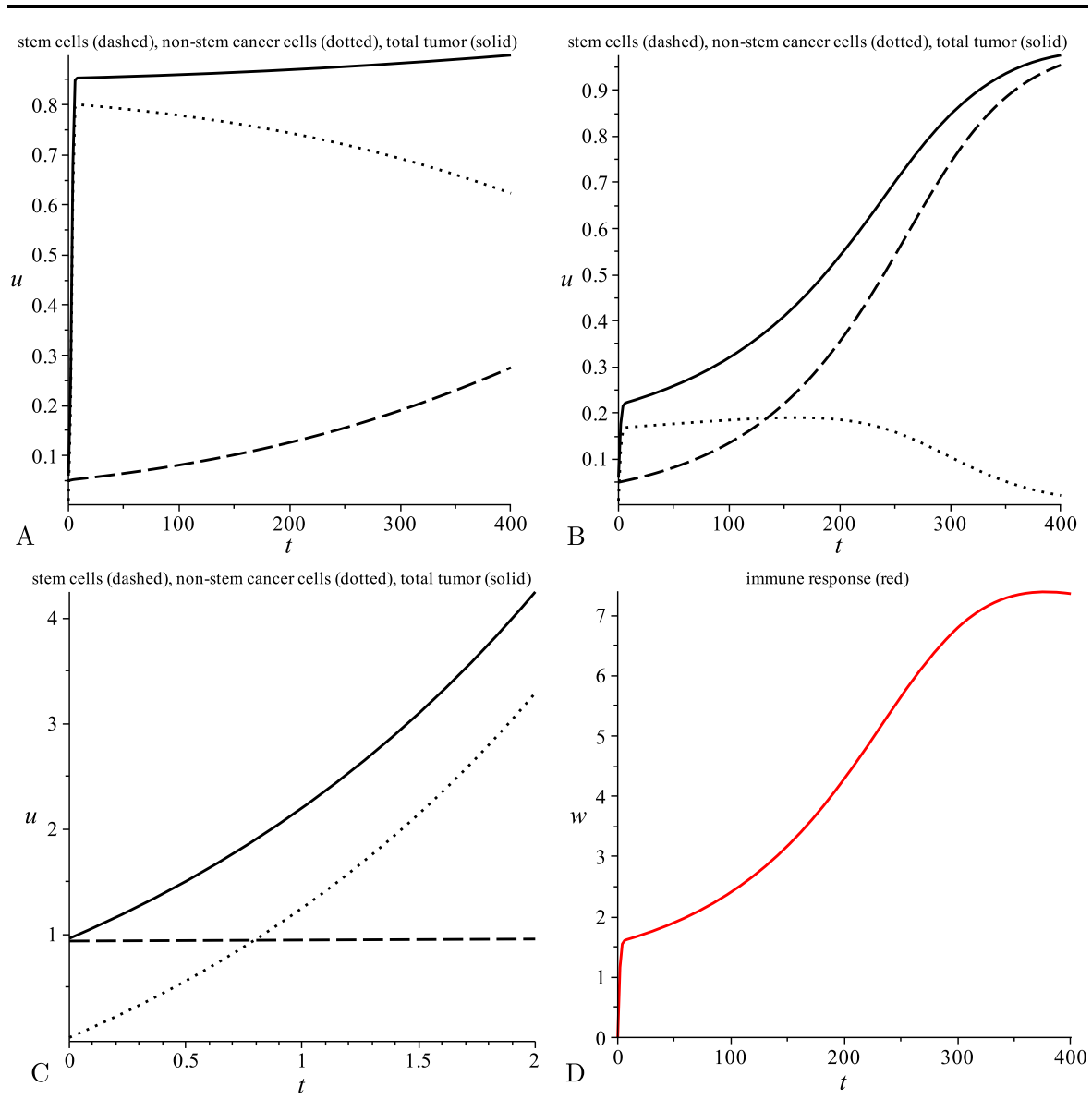

Fig. 9 A: Tumor growth without immune response, stem cells (dashed), tumor cells (dotted), total tumor (solid). B: Same initial condition as A, but now with immune response. D: immune response corresponding to B. C: Numerical reseeding experiment as discussed in remark 3. Parameters as described in the text

vitro or xenografted into immuno-deprived mice, for example, the selection force is removed and the intrinsic initial population ratios are revisited. Figure 9 on the bottom left shows a sample simulation of resecting an immuno-modulated tumor and in vitro expansion of the harvested populations. At the end of the simulation in the top right of Fig. 9, we harvest the tumor at $t=400$ days. On the bottom left of Fig. 9, we show how the tumor develops if left alone in a Petri dish $(w=0$, $k=1$ ). While CSC numbers remain almost unchanged, TC expand very quickly and dominate the population already after two days. This confirms experimental observations that cultured cancer stem cell probes contaminate very quickly. In light of these results, it seems impossible to estimate the CSC contents of a tumor if it is taken out of its environment and placed under different conditions.

4. Our model suggests that there is a significant difference between in vivo and in vitro tumor growth. For example, radiosensitivity measurements done in vitro might not reflect the response of the real tumor to radiation. 
5. Another interpretation which our model offers, is a hypothesis to explanation why it is so difficult to identify CSC and their frequency in a given tumor. Our work suggests that in some tumors almost all cells are CSC, whereas in other tumors they are indeed a very small subpopulation. In both cases, they are difficult to be distinguished from the tumor mass.

6. The model also suggests that, in some instances, it might be beneficial to reduce the immune response, thereby reducing the selection for cancer stem cells. As a result, the tumor will grow slower (for example, Fig. 9). If we inspect the tumor load at time $t=400$, then without immune response (left), we have a total tumor mass of $87 \%$ of the carrying capacity, while the tumor with immune response (right) shows an abundance of $97 \%$ of the carrying capacity.

7. It is discussed in the literature that the immune system can be both tumorinhibiting and tumor-promoting (Hanahan and Weinberg 2011). The mechanism discussed here can give a possible explanation of how the immune response promotes tumor growth. More detailed studies in this direction are certainly needed.

\section{Discussion}

Tumors are heterogeneous populations consisting of cells with different phenotype. A recently emerged phenotypic distinction is the so-called cancer stem cell hypothesis (Lapidot et al. 1994; Bonnet and Dick 1997; Al-Hajj et al. 2003; Dick 2003; Singh et al. 2003; Fioriti et al. 2008; Cammareri et al. 2008; Morrison and Kimble 2006; Reya et al. 2001). Cancer stem cells are immortal and can proliferate infinitely, whereas their nonstem progeny cancer cells have only a limited proliferation capacity before inevitable cell death. As tumors grow, evolving populations of cancer stem cells and non-stem cancer cells interact with each other in nonintuitive ways. We presented a mathematical model of the cancer stem cell hypothesis that is motivated by the paradoxical findings of an agent-based model (Enderling et al. 2009) that increased cell death may under certain conditions accelerate tumor growth-a phenomenon we call the tumor growth paradox. We developed a complex spatial integro-differential equation system based on reaction-birth process that mimics the rules of the agent-based model. For analytical convenience, we reduced this system to two ODEs, and were able to proof the existence of such tumor growth paradox in a spatially homogeneous system. Increased cell death initially reduced the overall tumor population, but in the long-term outgrows tumors with smaller cell death ratios. Analysis of the model further revealed that the ratio of cancer stem cells in a solid tumor can continuously change, and the only biologically realistic steady state of the system is a pure stem cell population. These findings augment to the ongoing discussions about frequency of cancer stem cells (Quintana et al. 2008). Tumors of the same patient and organ can present in the clinic with CSC fractions that differ by multiple orders of magnitude dependent on tumor history. Clinically apparent tumors are likely to have a long history before detection, and selection forces such as immune surveillance may additionally shape in vivo tumors to present highly enriched in CSC. The presented model provides first-order biologic mechanisms to explain the large variation in stem cell frequency reported in the literature (Visvader and 
Lindeman 2008) and complements arguments that any ratio of cancer stem cells to noncancer stem cells is possible (Johnston et al. 2010).

Acknowledgements The authors wish to thank Gerda de Vries and Jeff Bachman for fruitful discussions and remarks. The work of TH was supported by the Canadian NSERC. The work of HE was supported by the American Association for Cancer Research award number 08-40-02-ENDE (to HE) and the work of HE and PH was supported by the Office of Science (BER), US Department of Energy, under Award Number DE-SC0001434 (to PH).

\section{Appendix: Equivalence of Basic Stem Cell Models}

Here, we show that the three models for cancer stem cells that are illustrated in Fig. 2 are equivalent in the situation where the stem cell population is not declining. Let $U(t)$ and $V(t)$ denote the CSC and TC density at time $t$, and $k$ the rate of CSC division. For the purpose of demonstrating this equivalence, we ignore TC divisions. We first describe a hypothetical "complete model" (i) that has all three features, then demonstrate that dropping feature (ii) maintains model generality, while dropping feature (iii) also maintains generality provided parameters are chosen in the complete model such that the CSC compartment never decreases in time.

Complete Model We introduce the complete model that includes all three division fates described above. Let $\alpha_{1}$ denote the fraction of symmetric division, $\alpha_{2}$ the fraction of asymmetric division, and $\alpha_{3}$ the fraction of symmetric commitment events, with $\alpha_{1}+\alpha_{2}+\alpha_{3}=1$. A schematic is shown on the left in Fig. 2.

The change in cell populations due to CSC division events can then be described by:

$$
\begin{aligned}
& \dot{U}=\alpha_{1} k U-\alpha_{3} k U, \\
& \dot{V}=\alpha_{2} k U+2 \alpha_{3} k U .
\end{aligned}
$$

Invoking the identity $\alpha_{1}=1-\alpha_{2}-\alpha_{3}$, we obtain the system

$$
\begin{aligned}
& \dot{U}=\left(1-\alpha_{2}-2 \alpha_{3}\right) k U, \\
& \dot{V}=\left(\alpha_{2}+2 \alpha_{3}\right) k U,
\end{aligned}
$$

where $\alpha_{2}+2 \alpha_{3} \neq(0,1)$ and $\alpha_{2}+2 \alpha_{3}<1$ (or equivalently, $\alpha_{1}>\alpha_{3}$ ). The last condition arises from the assumption that the number of CSCs does not decrease in time.

No Symmetric Commitment Model This model assumes that CSC is a robust state that cannot be lost during mitosis (Enderling et al. 2009). Therefore, the dividing CSC always remains CSC, and the second daughter cell is either a CSC or a TC (Fig. 2). This model is the Complete Model with the additional condition of no chance of commitment, i.e., $\alpha_{3}=0$. From a simple inspection of Equation System (27) with $\alpha_{3}=0$, though, we see this model remains just as general as the Complete Model, since the leading coefficients on the right sides of the equations for $U$ and $V$ can range from 0 to 1 as before. 
No Asymmetric Division Model The model most often used in the literature ignores asymmetric CSC division (Ganguli and Puri 2006; Marciniak-Czochra et al. 2009; Wise et al. 2008). A mitotic CSC event either yields two CSC or two TC (Fig. 2). This model is the Complete Model with the additional condition of no chance of asymmetric division, i.e., $\alpha_{2}=0$. From a simple inspection of Equation System (27) with $\alpha_{2}=0$, though, we see this model remains just as general as the Complete Model, since the leading coefficients on the right sides of the equations for $U$ and $V$ can range from 0 to 1 as before.

In summary, we have shown that the "No Symmetric Commitment" and "No Asymmetric Division" models are individually equivalent to the "Complete Model," and so to each other. We therefore discuss a mathematical model that essentially exploits the "No Symmetric Commitment" model above, with the appreciation that it will not only provide analytic confirmation of the tumor growth paradox revealed by our agent-based studies (Enderling et al. 2009), but will simultaneously confirm the applicability of various sets of cell division rules we could alternatively have employed to build the model.

\section{References}

Al-Hajj, M., Wicha, M. S., Benito-Hernandez, A., Morrison, S. J., \& Clarke, M. F. (2003). Prospective identification of tumorigenic breast cancer cells. Proc. Natl. Acad. Sci. USA, 100(7), 3983-3988.

Alarcon, T., Owen, M. R., Byrne, H. M., \& Maini, P. K. (2006). Multiscale modelling of tumour growth and therapy: the influence of vessel normalisation on chemotherapy. Comput. Math. Methods Med., 7(2-3), 85-119.

Almog, N., Ma, L., Raychowdhury, R., Schwager, C., Erber, R., Short, S., Hlatky, L., Vajkoczy, P., Huber, P. E., Folkman, J., \& Abdollahi (2009). A transcriptional switch of dormant tumors to fast-growing angiogenic phenotype. Cancer Res., 69(3), 836-844.

Anderson, A. R. A., Weaver, A. M., Cummings, P. T., \& Quaranta, V. (2006). Tumor morphology and phenotypic evolution driven by selective pressure from the microenvironment. Cell, 127, 905-915.

Barcellos-Hoff, M. H. (2001). It takes a tissue to make a tumor: epigenetics, cancer and the microenvironment. J. Mammary Gland Biol. Neoplasia, 6(2), 213-221.

Bellomo, N., \& Delitala, M. (2008). From the mathematical kinetic, and stochastic game theory to modelling mutations, onset, progression and immune competition of cancer cells. Phys. Life Rev., 5, 183-206.

Bonnet, D., \& Dick, J. E. (1997). Human acute myeloid leukemia is organized as a hierarchy that originates from a primitive hematopoietic cell. Nat. Med., 3, 730-737.

Cammareri, P., Lombardo, Y., Francipane, M. G., et al. (2008). Isolation and culture of colon cancer stem cells. Methods Cell Biol., 86, 311-324.

Conde-Ramis, I., Drasdo, D., Anderson, A. R. A., \& Chaplain, M. A. J. (2008). Modelling the influence of the e-cadherin - beta-catenin pathway in cancer cell invasion: A multi-scale approach. Biophys. J., 95(1), 155-165.

de Pillis, L., \& Radunskaya, A. (2001). A mathematical tumor model with immune resistance and drug therapy: an optimal control approach. J. Theor. Med., 3, 79-100.

DeLisi, C., \& Rescigno, A. (1977). Immune surveillance and neoplasia-I: a minimal mathematical model. Bull. Math. Biol., 39(2), 201-221.

Dick, J. E. (2003). Breast cancer stem cells revealed. Proc. Natl. Acad. Sci. USA, 100(7), 3547-3549.

Dingli, D., \& Michor, F. (2006). Successful therapy must eradicate cancer stem cells. Stem Cells, 24(12), 2603-2610.

D'Onofrio, A. (2005). A general framework for modeling tumor-immune system competition and immunotherapy: Mathematical analysis and biomedical inferences. Physica D, 208, 220-235.

Dunn, G. P., Bruce, A. T., Ikeda, H., Old, L. J., \& Schreiber, R. D. (2002). Cancer immunoediting: from immunosurveillance to tumor escape. Nat. Immunol., 3(11), 991-998. 
Dunn, G. P., Old, L. J., \& Schreiber, R. D. (2004). The three E's of cancer immunoediting. Annu. Rev. Immunol., 22, 329-360.

Enderling, H., Alexander, N., Clark, E., et al. (2008). Dependence of invadopodia function on collagen fiber spacing and crosslinking: computational modeling and experimental evidence. Biophys. J., 95(5), 2203-2218.

Enderling, H., Anderson, A. R. A., Chaplain, M. A. J., Beheshti, A., Hlatky, L., \& Hahnfeldt, P. (2009). Paradoxical dependencies of tumor dormancy and progression on basic cell kinetics. Cancer Res., 69(22), 8814-8821.

Enderling, H., Hlatky, L., \& Hahnfeldt, P. (2009b). Migration rules: tumours are conglomerates of selfmetastases. Br. J. Cancer, 100(12), 1917-1925.

Fioriti, D., Mischitelli, M., Di Monaco, F., et al. (2008). Cancer stem cells in prostate adenocarcinoma: a target for new anticancer strategies. J. Cell. Physiol., 216(3), 571-575.

Folkman, J. (1971). Tumor angiogenesis: therapeutic implications. N. Engl. J. Med., 285(21), 1182-1186.

Folkman, J., \& Hanahan, D. (1991). Switch to the angiogenic phenotype during tumorigenesis. In Int. symp. Princess Takamatsu Cancer Res. Fund (Vol. 22, pp. 339-347).

Ganguli, R., \& Puri, I. K. (2006). Mathematical model for the cancer stem cell hypothesis. Cell Prolif., 39 , 3-14.

Gatenby, R. A., \& Gillies, R. J. (2008). A microenvironmental model of carcinogenesis. Nat. Rev. Cancer, $8(1), 56-61$.

Gevertz, J. L., \& Torquato, S. (2006). Modeling the effects of vasculature evolution on early brain tumor growth. J. Theor. Biol., 243(4), 517-531.

Greese, B. (2006). Development, analysis and application of a nonlinear integro-differential equation in the context of cancer growth. Diplomarbeit (MSc thesis), University of Greifswald, Germany, and University of Alberta.

Hahnfeldt, P., Panigrahy, D., Folkman, J., \& Hlatky, L. (1999). Tumor development under angiogenic signaling: a dynamical theory of tumor growth, treatment response, and postvascular dormancy. Cancer Res., 59(19), 4770-4775.

Hanahan, D., \& Weinberg, R. (2000). The hallmarks of cancer. Cell, 100(1), 57-70.

Hanahan, D., \& Weinberg, R. A. (2011). Hallmarks of cancer: The next generation. Cell, 144, 646-674.

Hek, G. (2010). Geometric singular perturbation theory in biological practice. J. Math. Biol., 60(3), 347386.

Johnston, M. D., Maini, P. K., Chapman, S. J., Edwards, C. M., \& Bodmer, W. F. (2010). On the proportion of cancer stem cells in a tumour. J. Theor. Biol., 266, 708-711.

Jones, C. K. R. T. (1994). Geometric singular perturbation theory. In J. Russell (Ed.), Dynamical systems, Montecatini Terme, Italy, 1994. Berlin: Springer. 2nd session of the Centro Internazionale Matematico Estivo (CIME).

Kim, M.-Y., Oskarsson, T., Acharrya, S., et al. (2009). Tumor self-seeding by circulating cancer cells. Cell, $139(7), 1315-1326$.

Kirschner, D., \& Panetta, J. C. (1998). Modeling immunotherapy of the tumor-immune interaction. J. Math. Biol., 37(3), 235-252.

Kuznetsov, V. (1987). Mathematical modeling of the development of dormant tumors and immune stimulation of their growth. Cybern. Syst. Anal., 23(4), 556-564.

Lapidot, T., Sirard, C., Murdoch, B., et al. (1994). A cell initiating human acute myeloid leukaemia after transplantation into scid mice. Nature, 367(6464), 645-648.

Liu, W., Hillen, T., \& Freedman, H. I. (2007). A mathematical model for M-phase specific chemotherapy including the G0-phase and immunoresponse. Math. Biosci. Eng., 4(2), 239-259.

Maitland, N. J., \& Colling, T. (2008). Prostate cancer stem cells: a new target for therapy. J. Clin. Oncol., 26(17), 2862-2870.

Mallet, D. G., \& De Pillis, L. G. (2006). A cellular automata model of tumor-immune system interactions. J. Theor. Biol., 239(3), 334-350.

Marciniak-Czochra, A., Stiehl, T., Ho, A. D., Jäger, W., \& Wagner, W. (2009). Modelling of asymmetric cell division in hematopoietic stem cells-regulation of self-renewal is essential for efficient repopulation. Stem Cells Dev., 18(3), 377-385.

Matzavinos, A., \& Chaplain, M. A. J. (2004). Travelling-wave analysis of a model of the immune response to cancer. C. R. Biol., 327, 995-1008.

Morrison, S. J., \& Kimble, J. (2006). Asymmetric and symmetric stem-cell divisions in development and cancer. Nature, 441(7097), 1068-1074.

Morton, C. I., Hlatky, L., Hahnfeldt, P., \& Enderling, H. (2011). Non-stem cancer cell kinetics modulate solid tumor progression. Theor. Biol. Med. Model., 8(1), 48. 
Naumov, G. N., Bender, E., Zurakowski, D., et al. (2006). A model of human tumor dormancy: an angiogenic switch from the nonangiogenic phenotype. J. Natl. Cancer Inst., 98(5), 316-325.

Norton, L. (2005). Conceptual and practical implications of breast tissue geometry: Toward a more effective, less toxic therapy. The Oncologist, 10(6), 370-381.

Prehn, R. T. (1972). The immune reaction as a stimulator of tumor growth. Science, 176(4031), $170-171$.

Prehn, R. T. (1991). The inhibition of tumor growth by tumor mass. Cancer Res., 51(1), $2-4$.

Quaranta, V., Rejniak, K. A., Gerlee, P., \& Anderson, A. R. A. (2008). Invasion emerges from cancer cell adaptation to competitive microenvironments: quantitative predictions from multiscale mathematical models. Semin. Cancer Biol., 18(5), 338-348.

Quaranta, V., Weaver, A. M., Cummings, P. T., \& Anderson, A. R. A. (2005). Mathematical modeling of cancer: the future of prognosis and treatment. Clin. Chim. Acta, 357(2), 173-179.

Quintana, E., Shackleton, M., Sabel, M. S., Fullen, D. R., Johnson, T. M., \& Morrison, S. J. (2008). Efficient tumour formation by single human melanoma cells. Nature, 456(7222), 593-598.

Reya, R., Morrison, S. J., Clarke, M. F., \& Weissman, I. L. (2001). Stem cells, cancer, and cancer stem cells. Nature, 414(6859), 105-111.

Ribba, B., Alarcon, T., Marron, K., Maini, P. K., \& Agur, Z. (2004). The use of hybrid cellular automaton models for improving cancer therapy. Lect. Notes Comput. Sci., 3305, 444-453.

Schatton, T., \& Frank, M. H. (2009). Antitumor immunity and cancer stem cells. Ann. N.Y. Acad. Sci., 1176, 154-169.

Sherratt, J. A., \& Nowak, M. A. (1992). Oncogenes, anti-oncogenes and the immune response to cancer: a mathematical model. Proc. Biol. Sci., 248(1323), 261-271.

Singh, S. K., Clarke, I. D., Terasaki, M., et al. (2004). Identification of human brain tumour initiating cells. Nature, 432(7015), 396-401.

Singh, S. K., Hawkins, C., Clarke, I. D., et al. (2003). Identification of a cancer stem cell in human brain tumors. Cancer Res., 63(18), 5821-5828.

Smallbone, K., Gatenby, R. A., Gillies, R. J., Maini, P. K., \& Gavaghan, D. J. (2007). Metabolic changes during carcinogenesis: potential impact on invasiveness. J. Theor. Biol., 244(4), 703-713.

Sole, R. V., Rodriguez-Caso, C., Diesboeck, T. S., \& Saldance, J. (2008). Cancer stem cells as the engine of unstable tumor progression. J. Theor. Biol., 253.

Teng, W. L., Swann, J. B., Koebel, C. M., Schreiber, R. D., \& Smyth, M. J. (2008). Immune-mediated dormancy: an equilibrium with cancer. J. Leukoc. Biol., 84(4), 988-993.

Todaro, M., Perez Alea, M., Di Stefano, A. B., et al. (2007). Colon cancer stem cells dictate tumor growth and resist cell death by production of interleukin-4. Cell Stem Cell, 1(4), 389-402.

Visvader, J., \& Lindeman, G. J. (2008). Cancer stem cells in solid tumours: accumulating evidence and unresolved questions. Nat. Rev. Cancer, 8(10), 755-768.

Wang, Z. A., \& Hillen, T. (2007). Classical solutions and pattern formation for a volume filling chemotaxis model. Chaos, 17, 037108. 13 pages.

Wise, S. M., Lowengrub, J. A., Frieboes, H. B., \& Cristini, V. (2008). Three-dimensional multispecies nonlinear tumor growth-I. J. Theor. Biol., 253, 524-543.

Youssefpour, H., Li, X., Lander, A. D., \& Lowengrub, J. S. (2012). Multispecies model of cell lineages and feedback control in solid tumors. J. Theor. Biol., 304, 39-59. 